\title{
The Analysis of Scheduling Algorithms in Microcellular 4G MIMO WINNER System
}

\author{
Paweł Sroka, Zbigniew Długaszewski
}

Original scientific paper

\begin{abstract}
In this paper impact of resource scheduling algorithms on the in the microcellular 4G environment has been analyzed. Among studied metrics were average user throughput, average cell's capacity and the quality of provided services, which has been defined as a percentage of users achieving given data rate with defined error rate. Well known memoryless scheduling algorithms such as Round Robin, MaxSNR and Fair Rate have been compared with the algorithms proposed for fading channels in [1], namely Proportional Fair and Score Based algorithms. The latter algorithms take into account both history of the scheduled users and channel conditions.

A simple modification has been proposed for all examined scheduling algorithms and its impact on the users and system performance has been analyzed. The above modification rely on a preselection of scheduled users, thus significantly reducing number of computations at the cost of some performance loss.

Presented results have been obtained for both uplink and downlink OFDMA transmission in the multipath MIMO channels in the Metropolitan Area scenario proposed within the WINNER project. Only the frequency adaptive transmission mode has been considered.
\end{abstract}

Index Terms - 4G, Manhattan grid, Metropolitan Area, OFDMA MIMO, scheduling.

\section{INTRODUCTION}

$\mathrm{O}$ ne of the main objectives of the IST WINNER project was to define a new radio interface for a ubiquitous radio system concept. The developed concept was a packetoriented, user-centric, always-best design targeting more than 100 Mbps maximum throughput for Metropolitan Area (MA) coverage.

Such a high throughput required application of MIMO technology in the physical layer and advanced MAC (Medium Access Control) layer processing. One of the main elements of the MAC layer is a resource scheduler, which includes scheduling for both frequency adaptive and non-frequency adaptive transmission. The integration of multiple access scheme into the MAC architecture allows large multi-user scheduling gains to be obtained with additional link

Manuscript received May 31, 2008 and revised August 29, 2008, and February 14,2009

This work has been performed in the framework of the IST project IST-4027756 WINNER 2, which was partly funded by the European Union.

P. Sroka and Z. Długaszewski are with the Poznan University of Technology, Chair of Wireless Communications Poznań, Poland (email: \{psroka, zdlugasz\}@et.put.poznan.pl). adaptation gain and large channel coding gain (using LDPC codes) for the frequency adaptive transmission. Additionally, short frame duration enables frequency-adaptive transmission at vehicular speeds and fast retransmissions, which allows obtaining reliable links also for services with delay constraints. In this paper only frequency adaptive transmission mode is studied.

The work presented in this paper is based on the MAC layer architecture developed within the WINNER project.

Radio transmission in the microcellular environment is influenced by multipath fading, users' mobility and interferences from neighboring cells. Thus, while some users may experience very good transmission conditions, others may be affected by deep fading or high interference level, which obstruct their transmission and may even rule out use of some resources. This complicates the task of the resource scheduling block and optimal allocation of the available radio resources.

Many solutions for the problem of resource scheduling were proposed in literature, which are following the rate adaptive approach aiming at maximizing the overall rate with a power constraint $[1,5,6]$. On the other hand, many works have been using Utility Theory to propose solutions for all the resource allocation algorithms, including also multi-carrier packet scheduling. Utility theory performs the optimization of a utility-pricing system, which is established based on the mapping of some performance criteria (e.g. rate, delay) or resource usage into the corresponding pricing values [7].

In this paper we present results of our work on the resource allocation for the OFDMA (Orthogonal Frequency Division Multiple Access) transmission in the MIMO microcellular environment. Analyzed metrics are the total average cell throughput and the distribution of the average user throughput. We are also checking if so-called satisfied users criterion (defined in the next section) is fulfilled.

The paper is organized as follows: in Section 2 selected scheduling algorithms are described. Later in Section 3 parameters and simulation assumptions of the WINNER radio interface are introduced. In Section 4 simulation results obtained for the uplink and downlink are presented and the performance of the examined scheduling algorithms is evaluated. Concluding remarks are presented in Section 5. 


\section{INVESTIGATED SCHEDULING ALGORITHMS}

Radio resource allocation is a multidimensional problem, which may be solved using algorithms whose implementation is very complex. In fact the allocation problem is in general not convex since the allocation variable is integer, thus in most cases the optimal solution can be found only evaluating all possible allocations. Therefore, most of the literature has been focused on the development of sub-optimal solutons that have a lower computational complexity but that still yield good results.

To analyse the impact of different long-term sharing objectives on user performance a flow level model has to be introduced. Let $n$ be the number of currently active users and $\phi_{i}$ the fraction of available resources allocated by the base station (BS) to user $i$, with:

$$
\sum_{i=1}^{n} \phi_{i}=1
$$

and the data rate of user $i$ defined as:

$$
\phi_{i} \times R_{i}
$$

where $R_{i}$ denotes the transmission rate of user $i$ when scheduled [1]. If the allocated resource block is relatively small each flow can be represented as a "fluid" data transfer with a rate given by (2) which varies as new flows are initiated and other complete.

Having defined the flow level model different scheduling strategies, such as fair time sharing, maximum rate sharing or fair rate sharing may be considered.

\section{A. Fair time slot sharing}

This strategy equalizes the number of resource block allocated to each user. Thus, the fraction of resources assigned to user $i$ may be defined as:

$$
\phi_{i}=\frac{1}{n}, \forall i=1, \ldots, n
$$

In this case the user performance depends on only two factors: the mean flow througput and the cell load [1].

This strategy of allocation may be realized using a simple Round Robin scheduler.

\section{B. Maximum rate sharing}

The maximum rate sharing, reffered to also as the maximum signal to noise ratio (MaxSNR) scheduling, is a strategy that selects always the users with the highest transmission rate. Hence, the ammount of resources allocated to user $i$ may be defined as [1]:

$$
\phi_{i}=1, \text { for } i=\arg \max _{j=1, \ldots, n} R_{j} .
$$

This strategy solves the scheduling problem by assigning the resource unit to the user that maximizes its gain, thus it maximizes the cell throughput. On the other hand such solution is extremely unfair, tending to privilege the users that are close to the BS and neglecting all the others.

\section{Fair rate sharing}

A fair strategy from a user perspective would be a one that equalizes the instantenous data rates. Thus, in view of (2) this can be realized by allocating the resources to users inversely proportional to their transmission rate. Such strategy is called the fair rate strategy and the scheduling process may be described by equation [1]:

$$
\phi_{i}=\frac{1 / R_{i}}{\sum_{j=1}^{n} 1 / R_{j}} .
$$

This strategy is the most fair one in terms of throughput, since all users will have approximately the same throughput. However, since this criterion maximizes the throughput of the worst user, it will provide low aggregate system throughput.

\section{Proportional Fair scheduling}

The Proportional Fair (PF) scheduler allocates the resource unit at time $t$ unit to user $i(t)$ with the highest transmission rate relative to its current average throughput, what can be represented using formula [1]:

$$
i(t)=\arg \max _{j=1, \ldots, n} \frac{R_{j}(t)}{T_{j}(t)},
$$

where the average throughput of user $j$ is typically evaluated through an exponentially smoothed average [1]:

$$
T_{j}(t)=\left(1-\frac{1}{t_{c}}\right) \times T_{j}(t-1)+\frac{1}{t_{c}} \times R_{j}(t) \mathrm{I}_{\{\mathrm{i}(\mathrm{t}-1)=\mathrm{j}\}} .
$$

The time constant $t_{c}$ determines the time-scale of the scheduler. A large value of $t_{c}$ gives the opportunity of waiting longer before scheduling a user when its channel quality peaks, thus better exploiting the multi-user diversity. On the other hand, if a packet delay tollerance of the applications is low the value of $t_{c}$ shall be relatively smaller [1].

The PF scheduler is an opportunistic scheduler, i.e. the most of resource units allocated to a user is correspond to local peaks of the feasible rate. Hence, this scheduling strategy provides a mechanism of sharing resources in a fair way ensuring good system performance at the flow level. 


\section{E. Score-Based scheduling}

The presented above PF scheduler's performance may suffer form assymetric fading or data rate constraints. Thus, a Score-Based (SB) scheduler has been developed which behaves like the PF scheduler in the ideal case. The SB algorithm allocates the resource unit at time $t$ unit to user $i(t)$ with the lowest score:

$$
i(t)=\arg \min _{j=1, \ldots, n} s_{j}(t),
$$

where the score $s_{j}(t)$ of user $\mathrm{j}$ at time $\mathrm{t}$ corresponds to the rank of its transmission rate $R_{j}(t)$ among the past values $\left\{R_{j}(t)\right.$, $\left.R_{j}(t-1), \ldots, R_{j}(t-W+1)\right\}$ observed in a time window of size $W$ [1]. Hence the score of user $i$ at time $t$ may be calculated as:

$$
S_{i}(t)=1+\sum_{l=1}^{W-1} 1_{\left\{R_{i}(t)<R_{i}(t-l)\right\}}+\sum_{l=1}^{W-1} 1_{\left\{R_{i}(t)=R_{i}(t-l)\right\}} X_{l},
$$

where $X_{l}$ are random variables on $\{0,1\}$ with probability $\mathrm{P}\left(X_{l}=0\right)=0.5[1]$.

The Score-Based scheduler selects a user when its transmission rate is high relative to its own rate statistics instead of selecting a user when its rate is high relative to its average throughput, which was the principle of the PF scheduler. Thus, it is not vunerable to the impact of assymetric fading or data rate constraints, keeping all of the advantages of the PF criterion.

\section{SYSTEM SETUP AND ITS PARAMETERS}

For the simulation of the microcellular $4 \mathrm{G}$ system one of the Metropolitan Area scenarios proposed in the WINNER project has been considered [2]. The metropolitan character of the enviroment has been represented using Manhattan grid of 121 (11 x 11) rectangular blocks of buildings and streets located around them [3]. [3] proposes two possible locations for the BSs: first one in the street crossings and the second one in the middle of the block of buildings between two blocks. The latter one was adopted within WINNER project for the Metropolitan Area scenario (see Fig. 1). Outdoor-toindoor transmission is not considered and all mobile terminals are uniformly distributed on the streets. Each mobile terminal (MT) is assigned to the BS offering the strongest signal at the MT location. The signal strength is calculated using path-loss models developed within the project [4].

Two distinct path-loss and channel models have been defined: one for the LOS (Line-of-Sight) and one for the NLOS (Non Line-of-Sight) channels. Terminals located on the streets perpendicular to the street in which the BS is located experience NLOS transmission conditions. Additionally, there exists probability of the NLOS conditions when both BS and MT are localized along the same street [4]. Both path-loss models include shadowing. Fast varying fading was not considered during assignment of the MTs to the BSs.

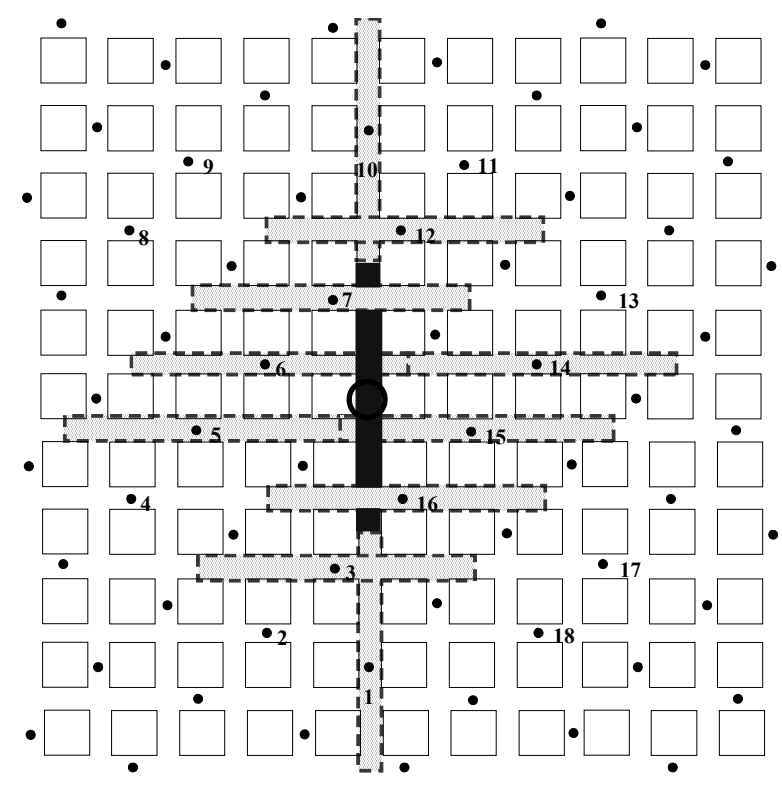

Fig. 1. Considered setup of Manhattan grid [3]

There are multiple cells in the microcellular Manhattan grid setup (see Fig. 1). Users and base stations located in other cells introduce intercell interference for the uplink and downlink transmission respectively. Such interference decreases signal to interference and noise ratio (SINR) and reduces achievable throughput, especially at the cell's edges. Rectangles shown in Fig. 1 represent approximate shape of cells. It was found out that about $90 \%$ of users located within such area were connected to the central BS. Some of users were located on perpendicular streets close to the BS, however, the propagation conditions caused by the lack of direct line of sight between MT and BS caused severe drop of signal power, and rewarded users located on the same street as the BS.

One does not have to consider interferences coming from all other BSs (for the downlink transmission) due to the path loss in the Manhattan grid. Considering BS located in the middle of Manhattan grid (circled one) one can consider only 18 neares BSs. It was shown in [8] that the strongest sources of interferences are the BSs located on the same street as MT. It was also shown, that without great loss of interfering power (and thus of the accuracy of the SNIR calculation) one can consider only 10 interfering BS. Similar analysis has been performed for the uplink transmission as well.

The considered system multiple access scheme was based for downstream and upstream on TDD OFDMA. The IFFT and FFT size was 2048, and the cyclic prefix had 128 samples. 1840 subcarriers were used for data transmission [2]. 15 consecutive OFDM symbols made up one downlink or uplink slot in the frame, 8 frames constituted one superframe. All resources available within one slot/frame were divided in the time and frequency domains into basic resource elements, called chunks. Signal bandwidth was set to $100 \mathrm{MHz}$, with the centre frequency of $3.95 \mathrm{GHz}$. A channel model developed in the WINNER project for Metropolitan Area 
environment has been used [4].

The uplink transmission was characterized by a lower power budget, thus one can expect that achievable throughputs will be lower than those obtained for the downlink transmission.

To improve the system performance, simple MIMO techniques have been employed. For the downlink transmission an eigenbeamforming scheme [9] has been used with 4 transmit antennas and 2 receive antennas. For the uplink transmission only simple Maximum Ratio Combining (MRC) [10] scheme with 2 transmit antennas and 4 receive antennas has been employed.

It was assumed that the positions of the MTs did not change during one superframe, hence there is no need to implement any handover scheme. In each frame only 8 users were served, however, to model the movement of the MTs every 10 superframes 40 or 20 different users were uniformly distributed within the microcell.

In each simulation run 10000 data frames (150 000 OFDM symbols) have been transmitted for both uplink and downlink. Users' throughput and overall cell's throughput have been calculated using previously obtained curves representing the throughput as a function of the signal to interference and noise ratio (SINR) for the assumed packet error ratio (PER).

In order to evaluate the QoS, the so-called satisfied user's criterion has been introduced. This criterion requires that at least 95 percent of users have been provided with $2 \mathrm{Mbit} / \mathrm{s}$ throughput with a given packet error rate.

It has been assumed that there is always data for transmission in the input buffer and there are no priority packets. The following resource scheduling algorithms have been investigated $[1,11]$ :

- $\quad$ Round Robin (RR)

- MaxSNR

- $\quad$ Fair Rate (FR)

- $\quad$ Proportional Fair (PF)

- $\quad$ Score Based (SB)

First three algorithms do not possess any memory. The Round Robin algorithm allocates each user the same amount of resources regardless of SINR. The MaxSNR scheduler allocates resources to those users who can use them in the most efficient way while the FR scheduler tries to guarantee the same throughput to all users. These algorithms are utilizing instantaneous values of the SINR values only. Calculation of SINR took into account SNR gains from the applied MIMO technique, link adaptation and LDPC coding.

The last two algorithms store history of each user's throughput and the current resource allocation depends on the channel conditions and average throughput each user has obtained so far. The Proportional Fair algorithm allocates resources to the users who can achieve the highest instantaneous data rate with respect to their average throughput while the Score Based algorithm allocates resources to the users who can transmit more in respect to the throughput statistics calculated in a certain time window [1].

For all of the algorithms mentioned above a modification based on the pre-selection of a small group of users for further scheduling using the Fair Time criterion has been proposed, where the same number of time slots was assigned to all users, thus resulting with a two-stage algorithms. These are referred to in the paper with the FT prefix, eg. FT/SB (Fair Time/Score-Based). The proposed modification reduces greatly the number of necessary computations in the scheduling process.

\section{Simulation Results}

To compare the proposed scheduling algorithms in terms of system performance and QoS, Monte Carlo simulations of the WINNER system have been performed. The investigated parameters were the average cell throughput and the user's throughput distribution function.

\section{A. Downlink}

The average cell throughput obtained for 40 users distributed uniformly within the cell is shown in Table I.

TABLE I

AVERAGE CELL THROUGHPUT DEPENDING ON THE EMPLOYED SCHEDULING ALGORITHM FOR DOWNLINK TRANSMISSION

\begin{tabular}{|c|c|}
\hline Scheduler & Throughput [Mbit/s] \\
\hline RR & 138 \\
FT/MaxSNR & 191 \\
MaxSNR & 192 \\
FR & 111 \\
FT/FR & 106 \\
FT/PF & 150 \\
PF & 152 \\
FT/SB & 143 \\
SB & 147 \\
\hline
\end{tabular}

The best scheduling algorithms in terms of the achieved average cell throughput are the ones using MaxSNR criterion for resources partitioning. The average cell throughput obtained in this case is higher than $190 \mathrm{Mbit} / \mathrm{s}$ for both FT/MaxSNR and MaxSNR schedulers. In this case the proposed modification of employing the Fair Time (FT) scheduling for pre-selection of users results in a substantial reduction of the number of required computations preserving the achieved high throughput. However, this algorithm allocates majority of the resources to a few users who experience good transmission conditions. Users located at the cell's edges and in the NLOS conditions have very slim chance to be served at all.

Slightly worse results in terms of achieved average cell throughput are obtained for the Proportional Fair (PF) and Score-Based (SB) schedulers. In this case the throughput value is around $140-150 \mathrm{Mbit} / \mathrm{s}$, but the proposed modification of users' pre-selection using Fair Time criterion results in throughput drop by 2-4 Mbit/s. 


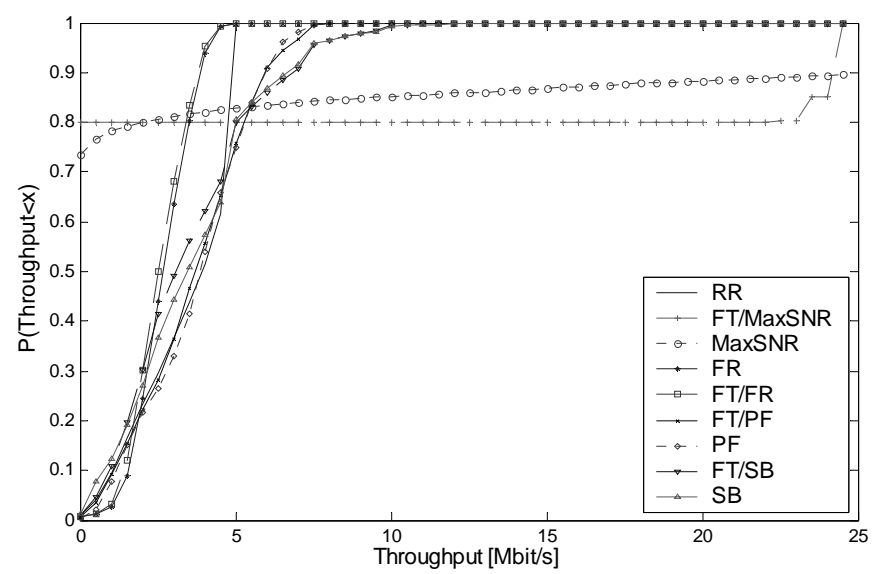

Fig. 2. User throughput distribution depending on the employed scheduling algorithm (40 users distributed in cell)

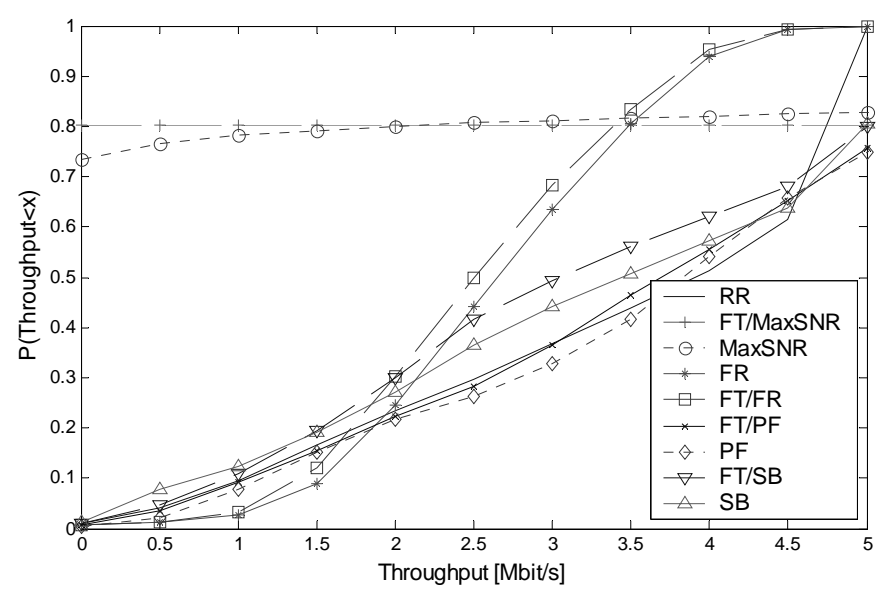

Fig. 3. User throughput distribution for selected scheduling algorithms (40 users distributed in cell)

The worst average cell throughput was obtained when using Fair Rate scheduling algorithms, as they tend to equalize the throughput for all users at each moment independently of the SINR values.

Fig. 2 and Fig. 3 present the users' throughput distribution depending on the used scheduling algorithm obtained with 40 users distributed uniformly within the cell. One can observe that the MaxSNR algorithms, which were the best in terms of the achieved average cell throughput, allocate all of the resources to only about 20 percent of the users. The highest "fairness" is achieved for the Fair Rate schedulers, but in this case the maximal offered throughput is lower than $4 \mathrm{Mbit} / \mathrm{s}$. Hence, the best solutions are the Proporional Fair and ScoreBased algorithms, as they allow to offer the throughput of at least $2 \mathrm{Mbit} / \mathrm{s}$ to $80 \%$ of the users with the maximum achievable rate of $10 \mathrm{Mbit} / \mathrm{s}$. Very interesting results were obtained when considering Round Robin scheduler, as the users' throughput distribution in this case is very similar to the one obtained for the PF schedulers, but the maximal achievable rate is lower.

The proposed modification of employing the Fair Time criterion for the pre-selection of 8 users yields very similar results as in the case of using more complex algorithms. Both FT/PF and FT/SB schedulers allow for almost identical users' throughput distribution as the "pure" PF and SB algorithms. Moreover, for the FT/MaxSNR scheduling the probability of achieving the througput higher than $2 \mathrm{Mbit} / \mathrm{s}$ is larger than in case of MaxSNR.

Similar results were obtained with only 20 users distributed uniformly within the cell. The achieved average cell throughput values were almost identical as in case of 40 users distributed in the cell. However, the users' throughput distribution may be different in this case as it is highly dependent on the number of users.

In Fig. 4 and Fig. 5 the user's throughput distribution depending on the used scheduling algorithm, when 20 users are distributed uniformly within the cell, is shown. The best results were obtained for the Proportional Fair and ScoreBased schedulers, as they allow to offer the throughput of at least $2 \mathrm{Mbit} / \mathrm{s}$ up to $95 \%$ of users, and the maximum achievable rate is $15 \mathrm{Mbit} / \mathrm{s}$.

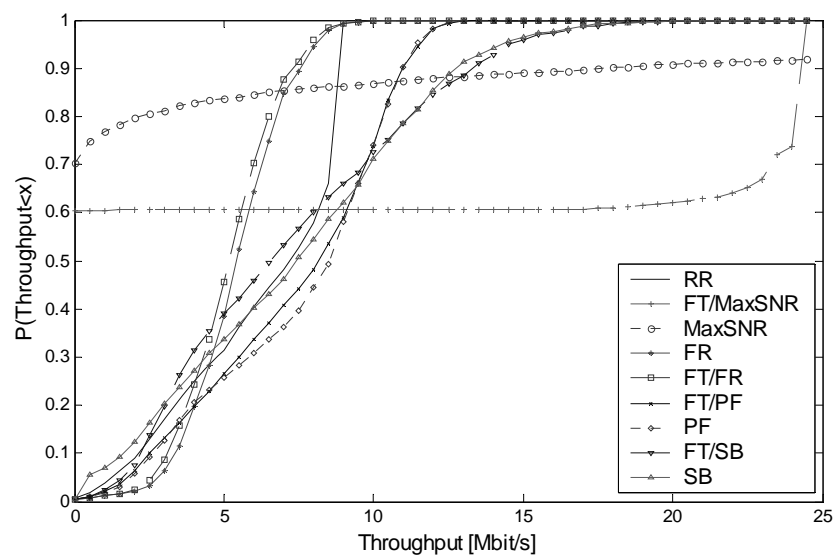

Fig. 4. User throughput distribution depending on the employed scheduling algorithm (20 users distributed in cell)

The performance of the proposed modified algorithms is almost identical as in the case of original schedulers. Moreover, the FT/SB algorithm provides the throughput of $2 \mathrm{Mbit} / \mathrm{s}$ for higher percentage of users than the original SB algorithm.

It can be clearly seen that reduction of the total number of users in the cell has allowed to increase each user's throughput and to fulfill the satisfied user's criterion.

\section{B. Uplink}

Identical analysis as presented above for the downlink, has been performed for the uplink transmission. Table 2 presents the average cell throughput obtained for 40 users distributed uniformly within the cell. 


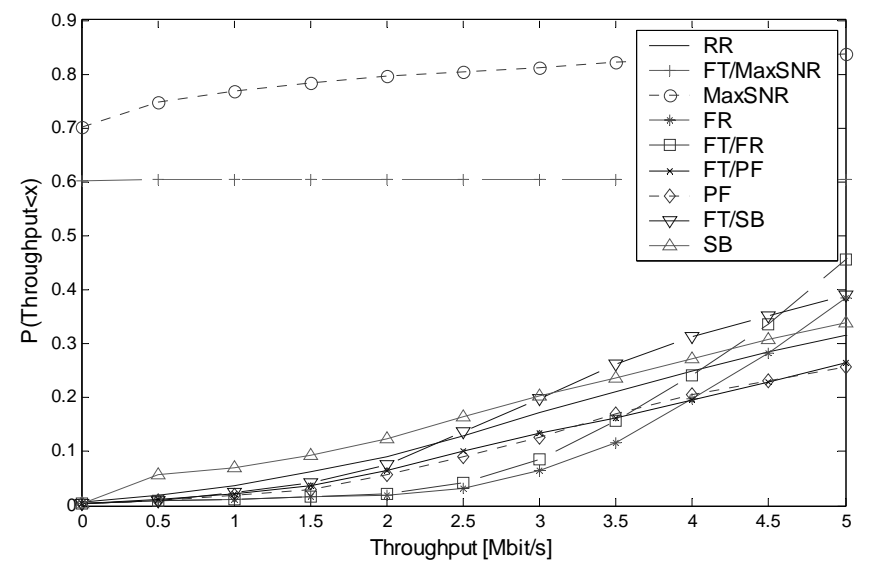

Fig. 5. User throughput distribution for selected scheduling algorithms (20 users distributed in cell)

TABLE II

AVERAGE CELL THROUGHPUT DEPENDING ON THE EMPLOYED SCHEDULING ALGORITHM FOR UPLINK TRANSMISSION

\begin{tabular}{|c|c|}
\hline Scheduler & Throughput [Mbit/s] \\
\hline RR & 93 \\
FT/MaxSNR & 182 \\
MaxSNR & 186 \\
FR & 50 \\
FT/FR & 43 \\
FT/PF & 122 \\
PF & 125 \\
FT/SB & 127 \\
SB & 130 \\
\hline
\end{tabular}

The best scheduling algorithms in terms of achieved average cell throughput are the ones using MaxSNR criterion for resources partitioning, and in this case the average cell throughput is around $180 \mathrm{Mbit} / \mathrm{s}$. The Proportional Fair (PF) and Score-Based (SB) schedulers are slightly worse providing average cell throughput around 120-130 Mbit/s. The proposed modification of users' pre-selection using Fair Time criterion results in throughput drop by 2-3 Mbit/s for both PF and SB schedulers. Fig. 6 and Fig. 7 present the users' throughput distribution depending on the used scheduling algorithm obtained with 40 users distributed uniformly within the cell for the uplink transmission. Similarly as in the downlink, the MaxSNR algorithms allocate all of the resources to only about 20 percent of the users, what makes them unsuitable for the microcellular environment.

The highest "fairness" is achieved for the Proporional Fair and Score-Based algorithms, as they allow to offer the throughput of at least $2 \mathrm{Mbit} / \mathrm{s}$ to more than 60 percent of the users with the maximum achievable rate up to $10 \mathrm{Mbit} / \mathrm{s}$. Both Fair Rate algorithms perform slightly worse than expected in terms of "fairness" due to the lower SINR values for all users caused by lower signal transmit power.

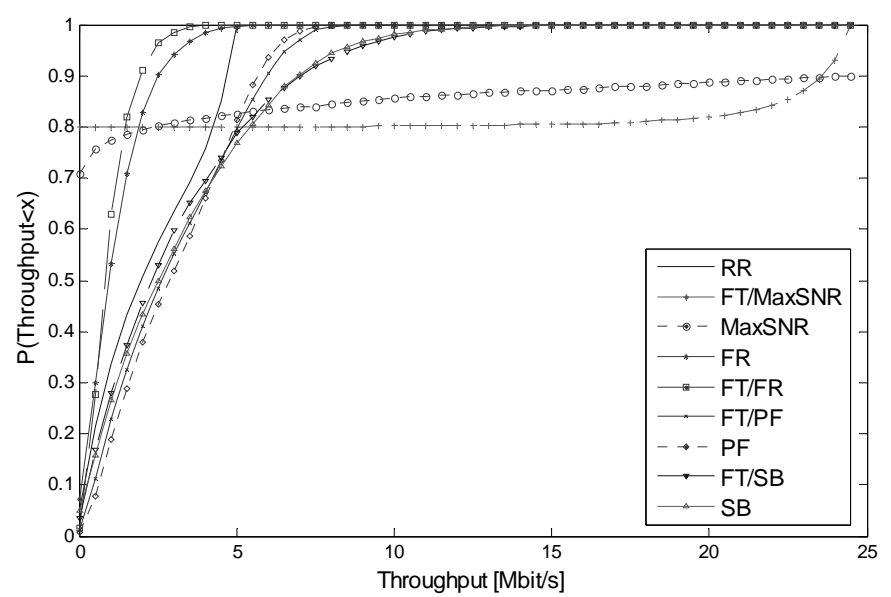

Fig. 6. User throughput distribution depending on the employed scheduling algorithm (40 users distributed in cell)

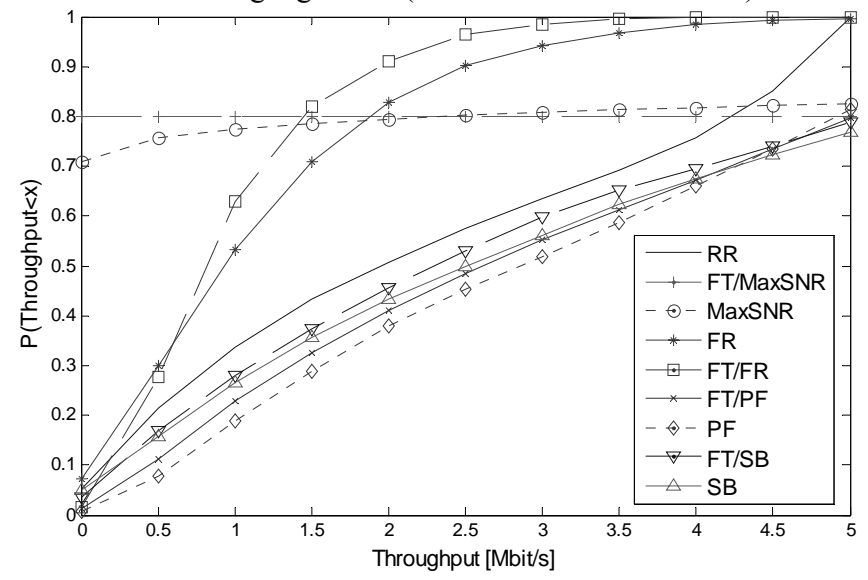

Fig. 7. User throughput distribution for selected scheduling algorithms (40 users distributed in cell)

The results obtained when considering the proposed modification of employing the Fair Time scheduler for preselection of 8 users are very similar to the ones in case of the original algorithms. Both FT/PF and FT/SB schedulers yield only slightly lower users' throughput in comparison with the "pure" PF and SB algorithms, but they result in significat reduction of the required number of computations. For the FT/MaxSNR scheduling the probability of achieving the throughput higher than $2 \mathrm{Mbit} / \mathrm{s}$ is the same or even higher than in case of the MaxSNR algorithm.

After reduction of the total number of users to 20 , the values of the average cell throughput are almost the same as in case of 40 users, the average throughput of user terminals has increased The user's throughput distribution for the uplink transmission, depending on the used scheduling algorithm when 20 users were distributed uniformly within the cell, is shown in Fig. 8 and Fig. 9.

The best results are obtained for the Proportional Fair (PF) and Score-Based (SB) scheduling algorithms, which allow to offer the throughput of at least $2 \mathrm{Mbit} / \mathrm{s}$ to more than 80 percent of the users. Fulfillment of the satisfied user criterion would require increasing power budget or application of a more advanced MIMO scheme, which might be difficult for the mobile terminal, implementation of some interference 


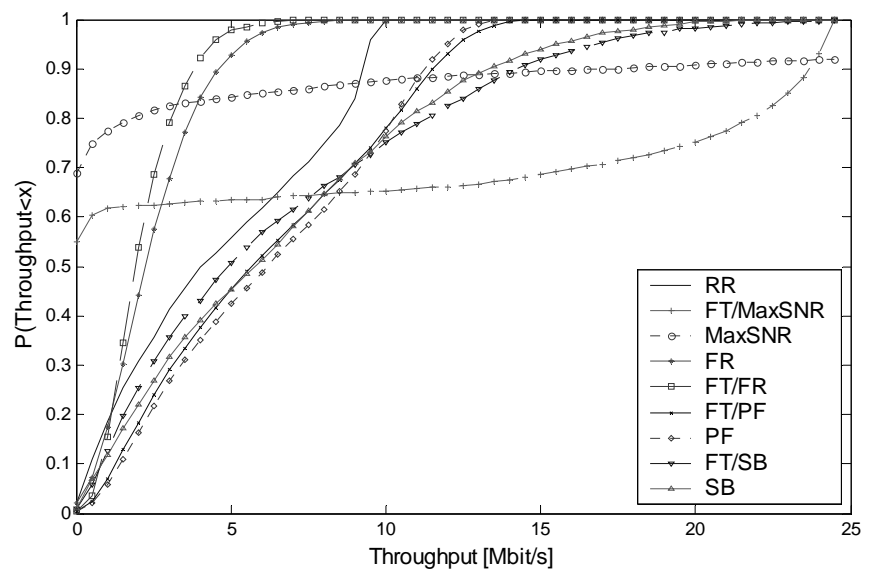

Fig. 8. User throughput distribution depending on the employed scheduling algorithm (20 users distributed in cell)

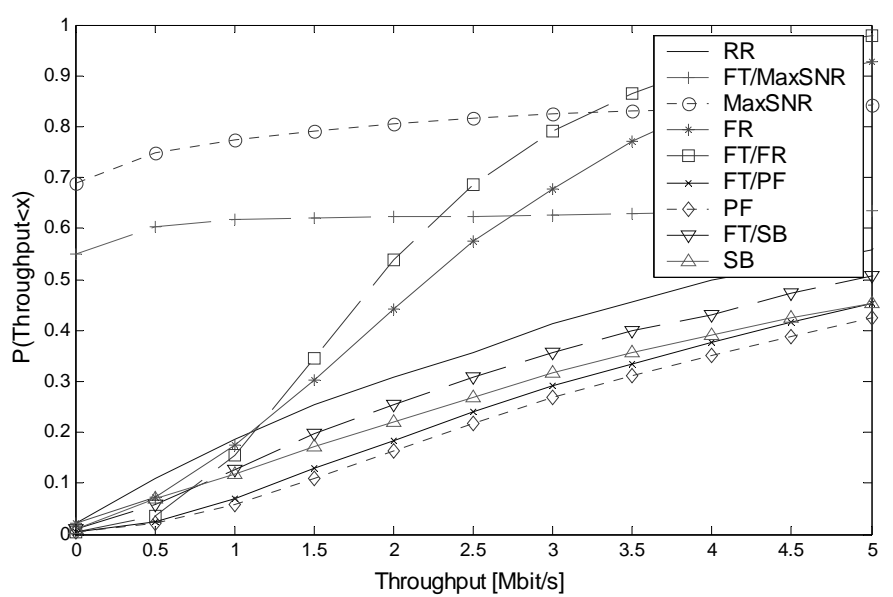

Fig. 9. User throughput distribution for selected scheduling algorithms (20 users distributed in cell)

suppression technique at the BS or further reduction of the number of users in cell.

The performance of the modified algorithms is almost as good as in the case of the original schedulers and requires fewer computations.

Concluding, the best candidates for resource schedulers for use in the considered microcellular $4 \mathrm{G}$ system are Proportional Fair and Score-Based algorithms, which provide great tradeoff between fairness and maximization of the achieved total cell's throughput. Moreover, it is worth considering the proposed modification to the original algorithms as the performance of the scheduler is almost the same, but the number of required computations is much lower.

\section{CONCLUSION}

In this paper the analysis of employment of various resource schedulers in $4 \mathrm{G}$ microcellular WINNER system has been presented. Careful investigation of the influence of the considered algorithms on the average cell throughput and the users' average throughput has been performed using Monte Carlo simulations.

It turned out that the Proportional Fair and Score-Based schedulers performed the best for such system. Although they provide lower total cell's throughput than the MaxSNR algorithms, they outperform all other solutions in terms of the average throughput offered to the mobile terminals. The use of the mentioned schedulers and considered MIMO techniques provides the throughput of at least $2 \mathrm{Mbit} / \mathrm{s}$ for up to 95 percent of the users for the downlink, and 80 percent of the users for the uplink transmission.

The proposed modification of users' pre-selection using the Fair Time algorithm, and further scheduling on a smaller group of users (eg. 8 users) reduces greatly the required number of computations, while keeping the average cell throughput and average offered user's throughput at almost the same level as the original PF and SB solutions.

The analysis shown in this paper shows that the best solution for $4 \mathrm{G}$ microcellular systems, such as WINNER system, may be either the Proportional Fair or Score-Based algorithm, however, a further improvement of system performance can be obtained by development of novel scheduling algorithms.

\section{REFERENCES}

[1] T. Bonald, "A Score-Based Opportunistic Scheduler for Fading Radio Channels", Proceedings of European Wireless, Barcelona, 2004.

[2] IST-2003-507581 WINNER D2.10, "Final report on identified RI key technologies, system concept, and their assesment", IST, 2005; available at http://www.istwinner.org/deliverables_older.html.

[3] ETSI TR $101 \quad 112$ v3.2.0, Universal Mobile Telecommunications System (UMTS); "Selection procedures for the choice of radio transmission technologies of the UMTS (UMTS 30.03 version 3.2.0)", ETSI, 1998.

[4] IST-2003-507581 WINNER D5.4, "Final Report on Link Level and System Level Channel Models", IST 2005; http://www.istwinner.org/deliverables_older.html.

[5] J. Jang and K. B. Lee, "Transmit power adaptation for multiuser OFDM systems," IEEE Journal on Selected Areas in Communications, vol. 21, no. 2, pp. 171-178, 2003.

[6] W. Rhee and J. M. Cioffi, "Increase in capacity of multiuser OFDMsystem using dynamic subchannel allocation," in Proc. IEEE 51st Vehicular Technology Conference - VTC Spring, 2000, pp. 1085-1089.

[7] G. Song and Y. G. Li, "Cross-layer optimization for OFDM wireless networks - part I: Theoretical framework and part II: Algorithmdevelopment," IEEE Transactions on Wireless Communications, vol. 4, no. 2, pp. 614-634,Mar. 2005.

[8] P. Sroka, Z. Długaszewski, ,Simulation of the metropolitan area cellular network using Manhattan grid scenario", Telecommunication Review + Telecommunication News, vol. 4, 2008, pp. 371-374, Poland, (in Polish). 
[9] J. Choi, S. R. Kim, I. K. Choi, "Eigenbeamforming with selection diversity for MIMO-OFDM downlink", 60th IEEE Vehicular Technology Conference VTC2004, vol. 3, Fall 2004, pp. 1806-1810.

[10] C.-H. Tse, K. -W. Yip, and T. -S. Ng, "Performance tradeoffs between maximum ratio transmission andswitched-transmit diversity", The 11th IEEE International Symposium on Personal, Indoor and Mobile Radio Communications PIMRC, vol. 2, London, 2000, pp. 1485-1489.

[11] A. Kumar, D. Manjunath. J. Kuri, "Communication Networking", Elsevier, 2004.

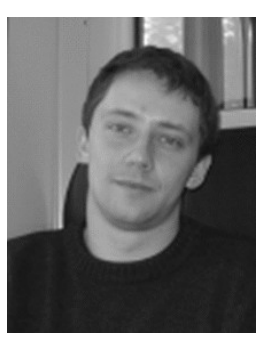

Pawel Sroka received his M.Sc. degree in Telecommunications from the Poznan University of Technology in 2004. Since 2004 he was employed at the Institute of Electronics and Telecommunications, and since 2007 at the Chair of Wireless Communications in the Faculty of Electronics and Telecommunications. Since 2004 he is the $\mathrm{PhD}$ student, and starting from the 1 October 2008 he works as the assistant at PUT.

His research interests covers the wide spectrum of wireless communications. Particularly, he is interested in multicarrier systems, in the area of radio resource management and cross-layer optimization.

He was involved in industrial and international research projects WINNER 2, WINNER+, and NEWCOM++.

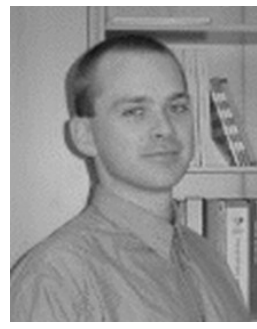

Zbigniew Długaszewski received his M.Sc. and $\mathrm{Ph} . \mathrm{D}$. degrees in Telecommunications from the Poznan University of Technology in 1999 and 2003 respectively. Currently he is employed as an assistant professor at the Chair of Wireless Communications at the Poznan University of Technology.

His research interests cover wireless multicarrier systems, synchronisation, coding theory, adaptation of link and system parameters, radio resource management and cross layer optimisation.

He was involved in many industrial and international research projects. 